\title{
UNSTEADY CFD ANALYSIS OF EROSION MECHANISM IN THE COOLANT CHANNELS OF A ROTATING GAS TURBINE BLADE
}

\author{
Domenico Borello, Davide Anielli, Franco Rispoli, Alessandro Salvagni, Paolo Venturini \\ Dipartimento di Ingegneria Meccanica e Aerospaziale, Sapienza Università di Roma \\ Via Eudossiana 18, Rome, Italy
}

\begin{abstract}
The two-phase flow in a rotating wedge mimicking the final portion of a blade turbine internal cooling channel is here presented and discussed focusing on unsteady motion and erosion mechanisms. The rotation axis is placed to properly reproduce a configuration with a very strong deviation $\left(90^{\circ}\right)$.

The flow field was modelled by using the well known $k-\varepsilon-\zeta$ $f$ unsteady-RANS model based on the elliptic-relaxation concept. The model was modified by some of the authors to take into account the influence of turbulence anisotropy as well as rotation. The model was applied to the well-established and fully validated $T$-Flow $S$ code.

A systematic comparison of rotating and non-rotating case was carried out to show the influence of Coriolis force on flow and erosion mechanisms.

The rotational effects strongly changed the flow behaviour within the channel, affecting both the unsteady flow and the particles trajectories. In the rotating case, there is no recirculation on the tip region; besides, position of the small recirculation regions above each pedestals change. These, and other minor effects, affect the particle motion thus resulting in a different erosion pattern.
\end{abstract}

\section{NOMENCLATURE}

Acronyms

CFD Computational Fluid Dynamics

LES Large Eddy Simulation

(U)RANS (Unsteady) Reynolds Averaged Navier Stokes

\section{SAS Scale Adaptive Simulation}

Ro Rotational number

Latin

$f \quad$ Elliptic relaxation parameter

$k \quad$ Turbulent kinetic energy

$\begin{array}{ll}\mathrm{L} & \text { Turbulent length scale } \\ P, G & \text { Production of k due to shear and to rotation } \\ S & \text { Shear stress invariant: } S=\sqrt{2 S_{i j} S_{i j}} \\ S t & \text { Strohual number } \\ S_{i j,} \Omega_{i j} & \text { Strain and rotation tensors } \\ u_{i} u_{j} & \text { Generic Reynolds Stress component } \\ v v & \text { Normal-to-the-wall Reynolds stress componen } \\ x, y, z & \text { Axial, radial, wall-normal coordinate } \\ \text { Greeks } & \\ \delta_{i j} & \text { Kronecker delta } \\ \varepsilon & \text { Dissipation rate of turbulent kinetic energy } \\ \varepsilon_{i j k} & \text { Levi-Civita symbol } \\ \zeta & v v / k \text { ratio } \\ v & \text { Kinematic viscosity } \\ v_{t} & \text { Turbulent kinematic viscosity } \\ \rho & \text { Density } \\ \sigma_{\mathrm{k}}, \sigma_{\varepsilon,}, \sigma_{\zeta} \text { Prandtl number for turbulent variables } \\ \Omega_{\mathrm{k}} \quad \text { Rotation vector }\end{array}$

\section{INTRODUCTION}

The internal cooling technique in gas turbine blades uses air spilled from the compressor and sent into internal channels to remove heat from the blade surface. To increase the heat transfer efficiency, generally turbulence promoters are present inside the cooling channel (protrusions, bumps, pedestals, pin fins). These objects have also a structural function. On the other hand, their presence increases the pressure losses and their number and shape must be carefully optimized. The flow becomes very complex, unsteady, and turbulent. A clear estimation of these phenomena is not easily obtained when using experimental measurement [1]. Furthermore, when 
considering turbines blades, the flow is subjected to rotation, strongly influencing streamlines and heat transfer. Influence of rotation on turbulent flows was well investigated in simple configurations (e.g. [2]). However, in complex configurations, detailed (LES) analysis of influence of rotation is not very well assessed and only few papers appear up to now [3], [4].

In real turbomachinery, the coolant flow is often seeded of solid particles crossing the compressor channels. Such dispersed particles consist of sand (or other particles) present in the injected air or they are generated by compressor blade erosion. The particles are drag by the carrier flow, impact on the solid surfaces thus producing fouling and/or erosion in the internal cooling channels. This provokes changes in the surface roughness, increasing losses and altering the heat transfer parameters. Furthermore, such regions are hardly accessible for inspections during their life cycle and then it is very difficult to estimate the growth of fouling and of erosion zones in operating conditions.

CFD represents a proper tool to face this problem provided that efficient models are employed. In turbomachinery applications, very simple RANS models were usually adopted. These are based on very empirical assumption aiming to obtain qualitative results at a reduced computational cost and with a good numerical stability [5], [6]. On the contrary, the adoption of physically sound models, able to recover the main turbulent features (unsteadiness, anisotropy, etc.) at a reasonable computational cost, can represent a valuable instrument for reducing costs for experimental tests and obtaining accurate clues for improving design methodology. Here we present an updated version of the unsteady, non-linear elliptic relaxation turbulence model (non linear $\mathrm{k}-\varepsilon-\zeta-\mathrm{f}$ URANS, [7]). This model is able to merge the basic capabilities of the elliptic-relaxation model proper treatment of the near-wall turbulence without the adoption of empirical, case dependent damping functions - with the adoption of non-linear approach to take into account the influence of turbulence anisotropy.

In a previous work [7] such model was used for obtaining an accurate unsteady solution of the flow inside the cooling channel of a non-rotating geometry, which reproduces the trailing edge cooling channels. Here we extended the model to include the influence of rotation, starting from the formulation of Petterson Reif at al., [8]. Finite volume code T-FlowS, currently developed in our research group at Sapienza was used for the present computations.

The potential of the present URANS formulation coupled with the numerical model well demonstrated in a previous paper [7]. We have to point out that we are able to reproduce unsteadiness due to large-scale motion (induced by the considered geometry), while turbulence fluctuations are out of the capabilities of any URANS solution. On the other hand, turbulence fluctuations are out from the scope of any engineering paper.
An in-house unsteady lagrangian code (P-Track [9],[10]) is used for tracking the particles motion, and Tabakoff approach is applied for predicting the erosion mechanism [11].

A comprehensive model for studying particles dispersion and erosion in rotating flows is then set up. Here, the model is applied to the analysis of the final portion of a rotating cooling channel reproducing the trailing edge region, where 7 pedestals were placed. Borello et al. [12] previously analyzed the flow and heat transfer in a similar test case when the flow is subjected to a solid body rotation by using an open source model. There, the well-known SAS model was used for flow prediction. In that case, one of the two side walls was heated.

The computational domain reproduces the experimental test case and comparisons with available results are presented. From the analysis of unsteady flow, we noticed the existence of periodical vortices probably related to the release of vortical structures from the pedestals.

In the next paragraph, we describe the mathematical models adopted for flow simulation, particles tracking and erosion prediction. Then, the present case will be described, and finally the flow, particle motion and erosion in the rotating trailing edge are discussed. Conclusions will close the paper.

\section{MATHEMATICAL MODELS}

\section{Flow model}

The mathematical model here adopted for describing the flow motion is an original, non-linear, incompressible $\mathrm{k}-\varepsilon-\zeta-\mathrm{f}$, low-Reynolds URANS equations set (1-6). The model was extended to take in account the influence of rotation

$$
\begin{aligned}
& \frac{D k}{D t}=P+G-\varepsilon+\frac{\partial}{\partial x_{j}}\left[\left(v+\frac{v_{t}}{\sigma_{k}}\right) \frac{\partial k}{\partial x_{j}}\right] \\
& \frac{D \varepsilon}{D t}=\left(C_{\varepsilon 1} P+C_{\varepsilon 3} G-C_{\varepsilon 2} \varepsilon\right) \frac{\varepsilon}{k}+\frac{\partial}{\partial x_{j}}\left(v+\frac{v_{t}}{\sigma_{\varepsilon}} \frac{\partial \varepsilon}{\partial x_{j}}\right) \\
& \frac{D \zeta}{D t}=f-\frac{\zeta}{k} P+\frac{\partial}{\partial x_{k}}\left[\left(v+\frac{v_{t}}{\sigma_{\zeta}}\right) \frac{\partial \zeta}{\partial x_{k}}\right] \\
& L^{2} \frac{\partial^{2} f}{\partial x_{j} \partial x_{j}}-f=\frac{C_{1}}{\tau}\left(\frac{\mathrm{v}^{2}}{k}-\frac{2}{3}\right)-C_{2} \frac{P}{k} \\
& \overline{u_{i} u_{j}}=\frac{2}{3} k \delta_{i j}-2 C_{\mu} \zeta k \tau_{1} S_{i j}- \\
& Y k \tau^{2}\left[C_{\mu 2}\left(S_{i k} \Omega_{k j}^{*}+S_{j k} \Omega_{k i}^{*}\right)-C_{\mu 3}\left(S_{i k} S_{k j}-\frac{1}{3}\left|S^{2}\right| \delta_{i j}\right)\right] \\
& \tau=\tau_{1}=\max \left[\min \left(\frac{k}{\varepsilon}, \frac{2}{3 C_{\mu 1} \zeta} \sqrt{\frac{3}{8|S|^{2}}}\right), 6\left(\frac{v}{\varepsilon}\right)^{0.5}\right]
\end{aligned}
$$

The expressions of the model coefficients are reported in [7].

The rotating flow is solved in a relative (rotating) frame of reference. Then Coriolis and centrifugal force must be accounted for in the momentum equation. However, in most 
URANS models, the equations of turbulent variables are not able to take into account the influence of rotation. This leads to large errors in the prediction of the flow behaviour. Here we applied a model correction to account for rotation. The correction was derived by authors starting from the formulation presented in [8] for a different URANS model.

Basic coefficients are reported in [8].

Following this approach, eddy viscosity coefficient $C_{\mu}$ was corrected with a rotation-dependent term obtaining $C_{\mu} *$, with the aim to "mimic" the behaviour of a second moment closure in rotating flows.

$C_{\mu}^{*}=C_{\mu} \frac{1+\alpha_{2}\left|\eta_{3}\right|+\alpha_{3} \eta_{3}}{1+\alpha_{4}\left|\eta_{3}\right|}\left(\sqrt{\frac{1+\alpha_{5} \eta_{1}}{1+\alpha_{5} \eta_{2}}}+\alpha_{1} \sqrt{\eta_{2}} \sqrt{\left|\eta_{3}\right|-\eta_{3}}\right)^{-1}$

where

$\eta_{1}=S_{i j} S_{i j} ; \eta_{2}=W_{i j}^{*} W_{i j}^{*} ; \eta_{3}=\eta_{1}-\eta_{2}$

$W_{i j}^{*}=T\left(W_{i j}+2 C_{\omega} \varepsilon_{j i k} \Omega_{k}\right)$

Comparisons with DNS [13] gave satisfactory results (see below).

\section{Particle transport and erosion}

In the present study particles are individually tracked in a Lagrangian framework. In turbomachinery applications the fraction of solid particles entrained by the flow is usually small $\left(<10^{-6}\right)$, thus inter-particle collisions are very rare and the effect of particles on the fluid motion is negligible ([14], [15]). In this case a one-way coupling approach can be used, that is particle motion is affected only by flow motion, but flow is not affected by the particles.

Newton's Second law (in the form of BBO equation [14]) is solved to compute the motion of each particle. Since the average size of particles involved in turbomachinery flows is few $\mu \mathrm{m}$ ([16], [17]) the only relevant forces acting on particles are drag and gravity [18], [19]. In the present study, gravity is neglected since the mass of simulated particles and the domain size are relatively small, thus its effect on particle motion is hardly appreciable during the average time interval a particle takes to pass through the domain. Therefore, indicating with $\vec{v}$ and $\vec{u}$ respectively particle and gas velocities, the BBO equation writes

$\rho_{p} \frac{d \vec{v}}{d t}=-\frac{3}{4 d_{p}} \rho_{f} C_{D}(\vec{v}-\vec{u})|\vec{v}-\vec{u}|$

In order to account for the relative reference framework, the BBO equation (8) was modified adding the Coriolis and centrifugal forces.

Particles are assumed to be spherical, non-rotating and nonreacting.

Erosion is a very complex phenomenon since it involves particle impact velocity and angle, particle shape, and material properties of both particles and target surface. Developing a theoretical model is extremely difficult. According to their experiments, Tabackoff et al. [11] developed an empirical correlation for predicting the erosion per unit mass of impacting particles (ER, in $\mathrm{mg} / \mathrm{g}$ ) of different materials. Their model accounts for the main parameters affecting erosion.

$$
E R=K_{1} f_{\alpha}|\vec{v}|^{2} \cos ^{2} \alpha\left(1-R_{T}^{2}\right)+f_{i}
$$

where

$R_{T}=1-0.0061|\vec{v}| \sin \alpha$

$f_{\alpha}=\left\{1+K_{C}\left[K_{12} \sin \left(90 \alpha / \alpha_{0}\right)\right]\right\}^{2}$

$f_{i}=K_{2}(|\vec{v}| \sin \alpha)^{4}$

$K_{1}, K_{2}$ and $K_{C}$ are empirical constants determined by Tabackoff and coworkers [11], and are function of material properties of particles and target surface. In the present work it is assumed that the channel walls are made of stainless steel. Accordingly, Table 1 shows the material coefficients. Notably, Tabakoff's correlation prescribed an angle of maximum erosion $\alpha_{0}$ depending on target material; for stainless steel it is set equal to 30 degrees.

Table 1. Erosion model coefficients.

\begin{tabular}{ccc}
\multicolumn{2}{c}{ Table 1. Erosion model coefficients. } \\
\hline$K_{C}$ & $\begin{array}{c}\alpha \leq 3 \alpha_{0} \\
\alpha>3 \alpha_{0}\end{array}$ & 1 \\
& & $1.505101 \cdot 10^{-06}$ \\
\hline$K_{1}$ & & $2.96077 \cdot 10^{-01}$ \\
\hline$K_{l 2}$ & & $5.0 \cdot 10^{-12}$ \\
\hline$K_{2}$ & &
\end{tabular}
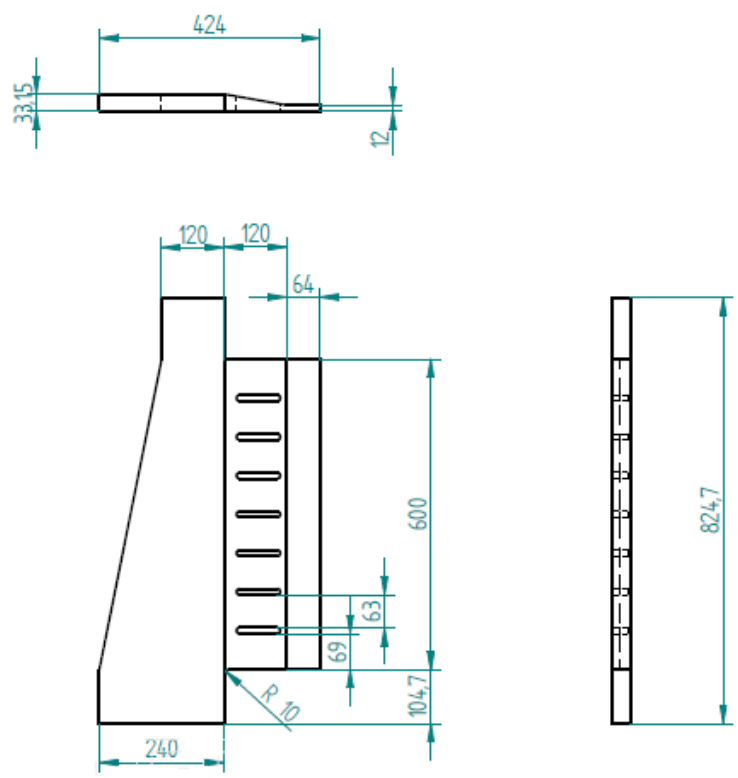

Figure 1. Size (in mm) of the wedge-shaped channel used in experiments [1]. 


\section{COMPUTATIONAL DETAILS}

Present configuration reproduces the one used in the experiments carried out at University of Florence [1], see also bibliography reported in [12].

A rotating wedge-shaped channel, reproducing the last region of the internal cooling channel, close to the turbine blade trailing edge was studied (see Figure 1 and Figure 2). The flow direction rotates of $90^{\circ}$. Furthermore, 7 pedestals were introduced (numbered in the Figure). The shape of the wedge in the $x-y$ direction is shown in the right part of the Figure. It is possible to see that, after the $90^{\circ}$ turning, the channel becomes convergent leading to a strong flow acceleration, forcing a strong streamlines curvature.

In the experimental test, the tip region (at maximum z) can be open or closed. Here we consider only the closed configuration. The Reynolds number, based on the hydraulic diameter, bulk inlet velocity and air property is equal to 20,000.

To detect the occurrence of large unsteady motions, in a previous paper focused on the non-rotating configuration [20], we analyzed the velocity fluctuations in several monitoring points. We noticed that periodic fluctuations were present in all the 19 monitoring points there considered, all having a period of $\mathrm{St}=0.22$. To investigate the existence of similar fluctuations in the rotating case, also in this case unsteady RANS simulation was carried out.

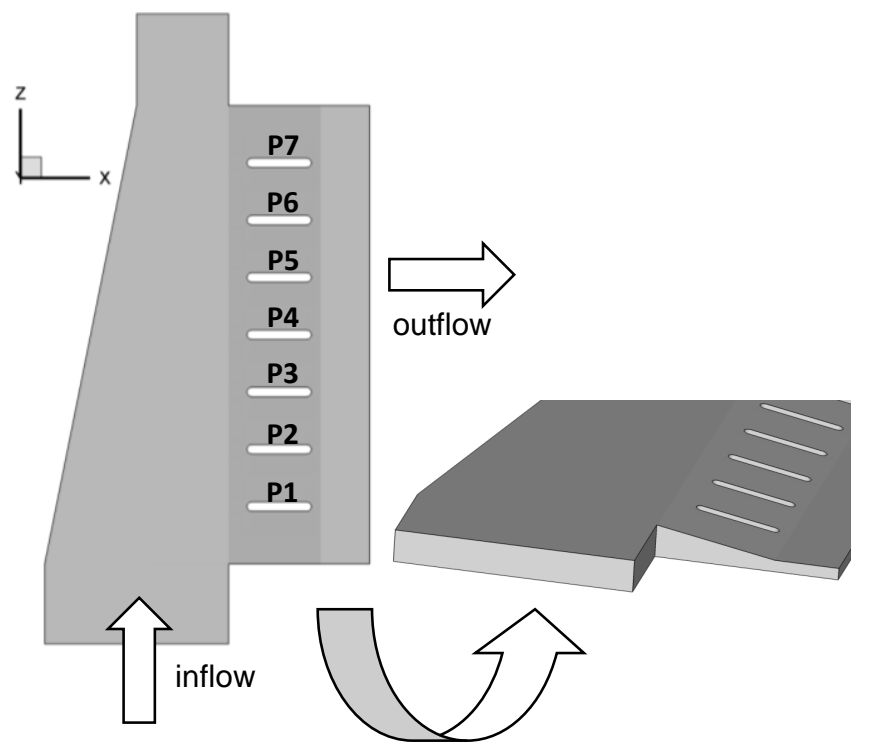

Figure 2. Geometry of the cooling channel.

Figure 3 shows the computational grid here adopted. It has 4.5 million of hexahedral cells. The description of the pedestal region discretisation is shown in top-right region. As for the discretisation of the $90^{\circ}$ corner in the red ellipse, a preliminary test was carried out reproducing the CAD profile. This geometry led to an abnormal flow deviation and in a consequent not-realistic prediction of turbulent variables in the region and, finally in a rapid divergence of the solution. Then a small rounded edge was introduced to model such region (see the blow-up in Figure 3-right down).

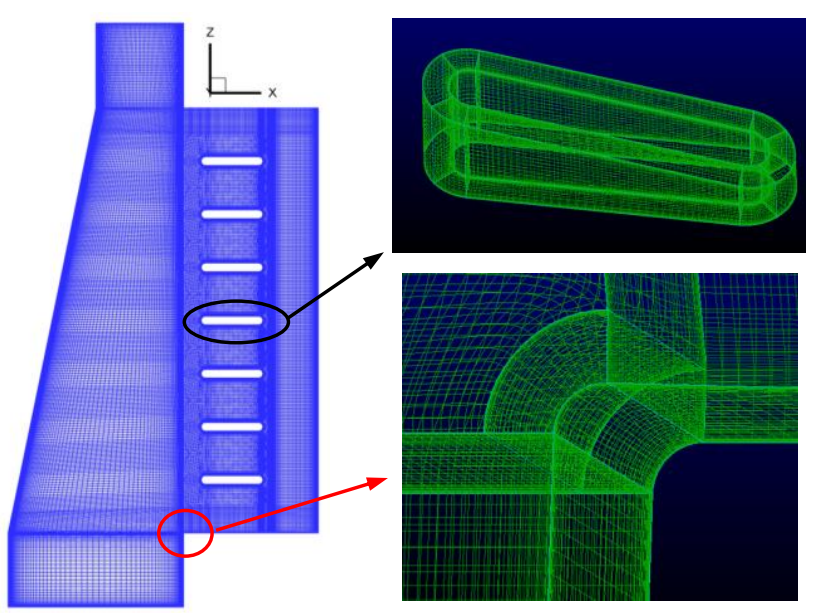

Figure 3. Computational mesh: whole domain and detailed. views of the pedestal region and the rounded angle.

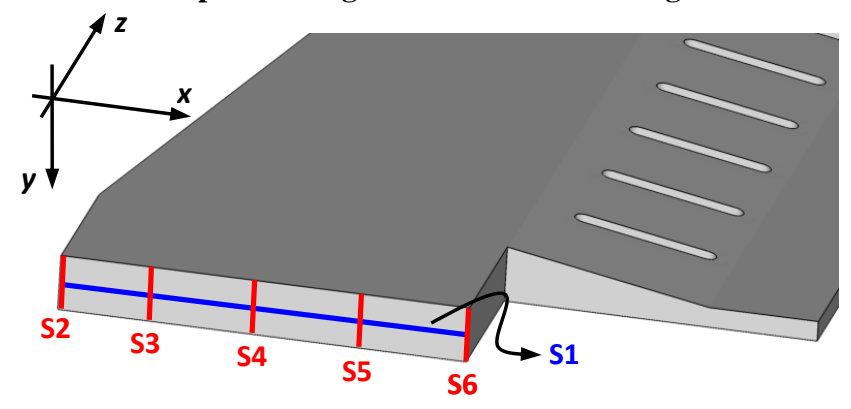

Figure 4. Sections where particles enter the domain.

The domain is rotating around $y$-axis with a $\mathrm{Ro}=0.275$, being Ro

$R o=\frac{\Omega D_{h}}{U_{b}}$

The boundary conditions are reported in Table 2 .

The Reynolds number based on the hydraulic diameter of the inlet section, air properties and inflow velocity, is equal to 20,000 . The inlet turbulent intensity was equal to $3 \%$ and the ratio of turbulent and molecular viscosity at the inlet was set equal to 10.0.

Table 2. Boundary conditions.

\begin{tabular}{|l|l|l|}
\hline Inlet & Outlet & Wall \\
\hline $\mathrm{U}=5.3 \mathrm{~m} / \mathrm{s}$ & Zero-gradient & $\begin{array}{l}\text { No-slip, } \\
\text { adiabatic }\end{array}$ \\
\hline
\end{tabular}

The Navier-Stokes equation system (continuity and momentum equations) was solved by adopting SIMPLE coupled with SMART algorithm for controlling flow instabilities and BiCG-Stab linear solver.

The unsteady field was computed by using a second-order accurate scheme. Time step was set to have the CFL always smaller than 1.0 in all the computational cells. 


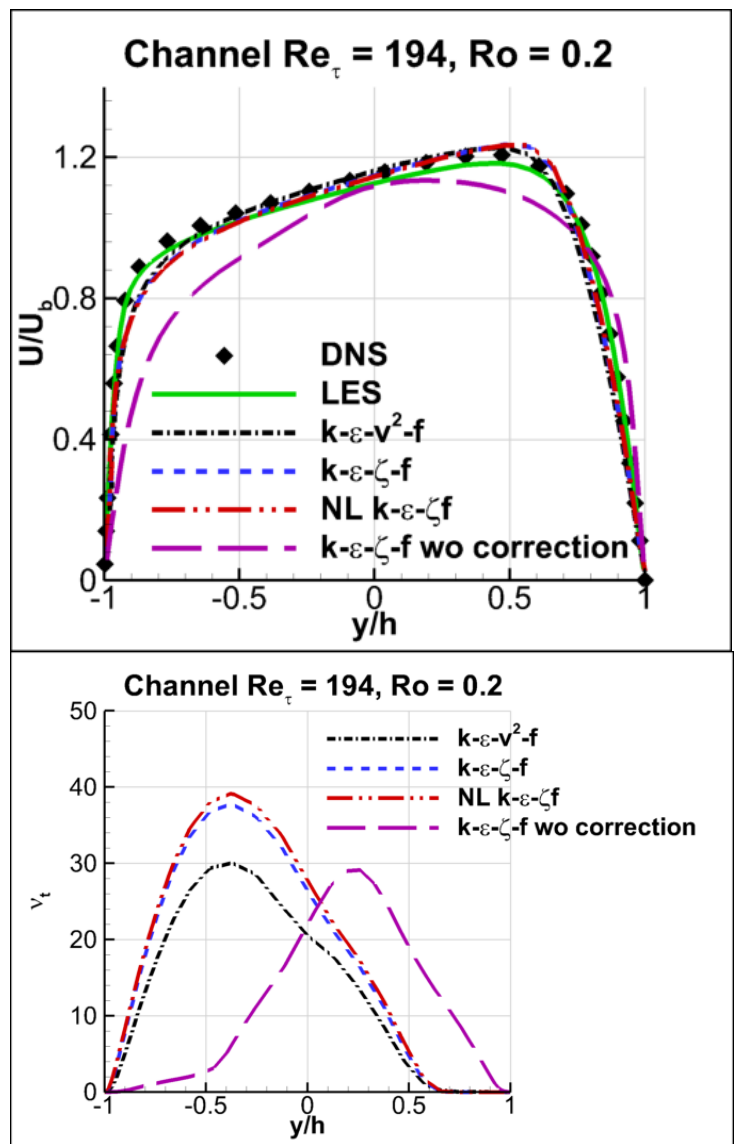

Figure 5. Rotating channel: velocity profile (top) and turbulent viscosity profile (bottom).

$P$-Track code is an in-house Finite-Element-based code for multiphase flow simulations developed by the authors at Sapienza Università di Roma and applied in several previous studies (see for example [7], [20]-[23]). Here it is used to predict particle transport and erosion.

As in erosion prediction realistic particle size and velocity are very important to have credible results, in particles simulation the size of the cooling channel is scaled down to a more realistic size (i.e., $5 \mathrm{~cm}$ in $z$ direction), and the velocity scaled up to maintain the same Reynolds number (resulting in an inlet velocity of about $87.0 \mathrm{~m} / \mathrm{s}$ ). Besides, in order to account for the unsteadiness of the flow (see RESULT section), the flow characteristic period has been divided into 24 different realizations, which have then been used as different flow field by $P$-Track code at the proper time instant.

With the aim of studying the effect on particle motion of different turbulent structures occurring within the channel, particles enter the domain from some selected cells, as already done in the non-rotating study [20]. The selected cells are distributed on six lines at the inlet surface (Figure 4), named S1-S6. 80 spherical, non-rotating and non-reacting particles, having the same flow velocity at the inlet, are seeded in each starting cell, and at each new realization, thus accounting for the effect of different flow realizations on particle dispersion. This seeding strategy results in simulating more than 382,000 particles, which is a sufficiently large number of particles to obtain statistically meaningful results. Moreover, erosion rate is normalized with reference to the maximum value of the rotation case.

Particle properties used in the simulations are reported in Table 3

Table 3: Particles properties.

\begin{tabular}{ccc}
\hline$d_{p}(\mu \mathrm{m})$ & $\rho_{p}\left(\mathrm{~kg} / \mathrm{m}^{3}\right)$ & $U_{\text {in }}(\mathrm{m} / \mathrm{s})$ \\
\hline 5 & 1500 & 87.0 \\
\hline
\end{tabular}

\section{RESULTS}

\section{Flow Motion}

The main topic of the present contribution is to put in evidence the influence of rotation on both flow and particle motion. Then, a systematic comparison with the non rotating configuration is carried out with reference to several planes and their intersection.

For the rotating case, we had no experimental results. However, accuracy of the model was assessed in a previous paper focused on the non-rotating case [7].

As a preliminary assessment of the developed turbulence model, in Figure 5 the velocity profile in a rotating channel flow with a $\operatorname{Re}_{\tau}=194$ and $\mathrm{Ro}=0.2$ is shown. Velocity profile in the bulk flow should have an almost linear profile with a slope equal to Ro [12].

In Figure 5, all the models except one (purple line) were sensitized to rotation and are able to correctly reproduce the velocity profile. The influence of the model is clearly shown when analysing the eddy viscosity distribution.

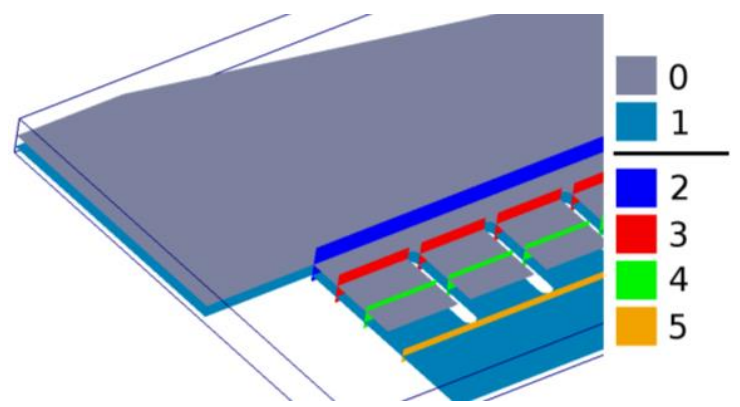

Figure 6. Reference planes with colour keys. Reference lines cited in the text correspond to the intersection of these planes (e.g., line 03 is the intersection of planes 0 and 3 ).

The correction introduced changes the shape of the eddy viscosity distribution moving the peak on the opposite site with reference to the unchanged configuration. It is interesting to note that linear and non-linear $\zeta$-f models show similar results.

As for the analysis of the present configuration, the sections used in experimental test were indicated in Figure 6. Planes 0 and 1 are placed at $50 \%$ and $25 \%$ of the blade height in $y$ normal direction (cft. Figure 1). Sections 2-5 are placed in $\mathrm{x}$ normal direction as shown in Figure 6. 

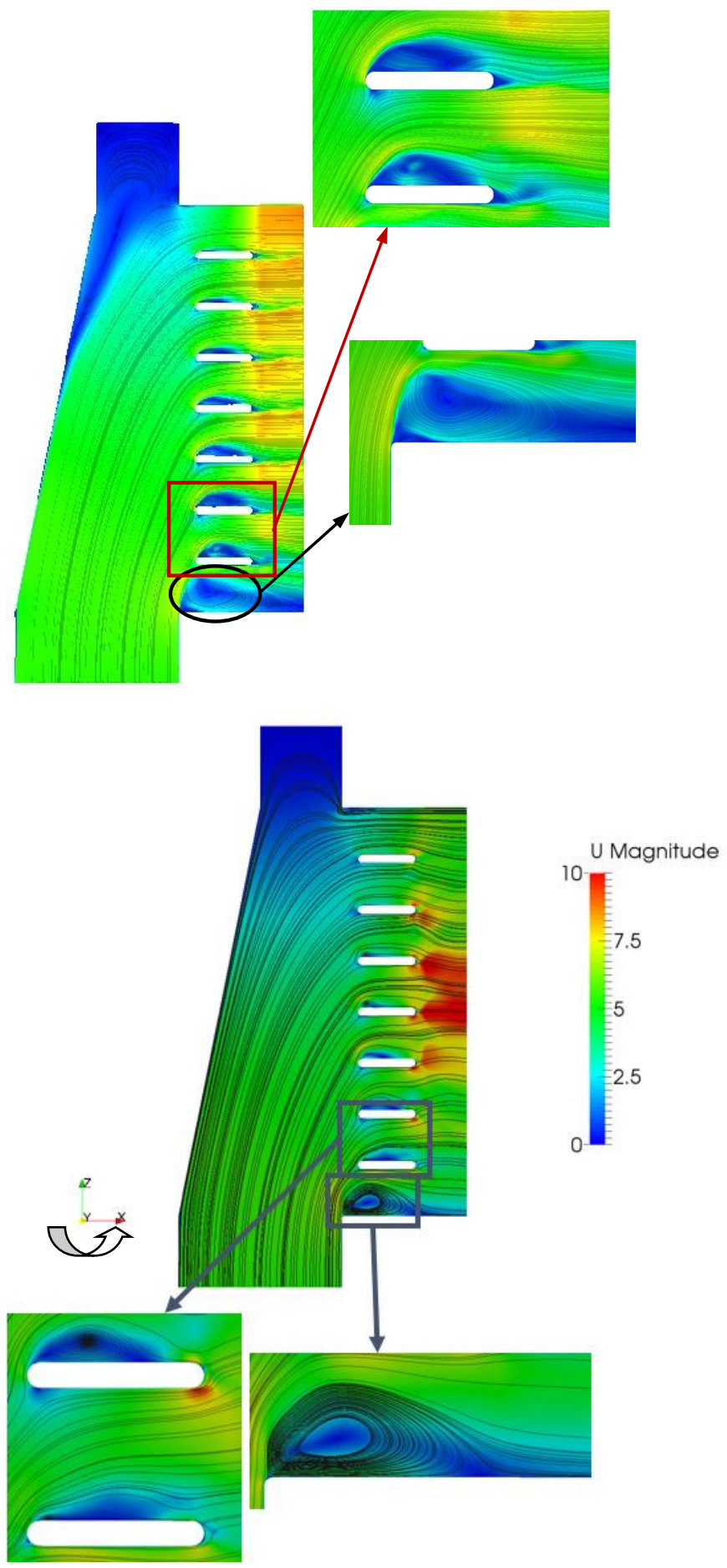

Figure 7. Velocity magnitude plot and streamlines on Plane 1 for the non-rotating (top) and rotating (bottom) configuration.

To obtain an overall evaluation of the rotation influence on the velocity field, in Figure 7 the velocity magnitude and streamlines on plane 1 are reported. On this plane, the solid body rotation causes an anticipated, stronger streamlines deviation. This leads to the destruction of the recirculation zone present at the tip region. Furthermore, the recirculation bubbles placed downstream of the $90^{\circ}$ corner and over the pedestals reduced their extension and the blockage of fluid passage sections between two successive pedestals.

Finally, the velocity distribution in the exit region is less uniformly distributed showing a strong mass flow rate downstream the pedestals 3-5.
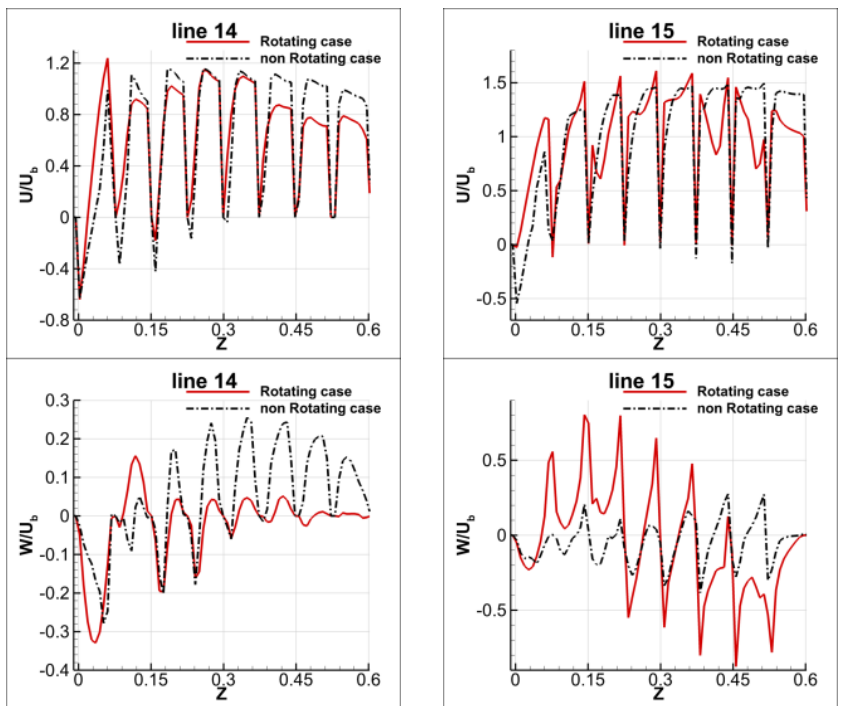

Figure 8. Comparisons of velocity components profiles for two different lines obtained by the intersection of plane 1 with planes 4 and 5 .

In Figure 8, the velocity profiles along lines obtained by the intersection of plane 1 with planes 4 (mid of the pedestals) and plane 5 (downstream from the pedestals) are shown. Comparison with non-rotating case is reported. The U velocity profile (directed as the outflow direction) confirms the findings previously discussed: larger velocity between pedestals 3-5 and above the $90^{\circ}$ corner. $\mathrm{W}$ velocity is strongly damped in line 14 , especially when moving towards the pedestals at higher values, due to the strong forcing induced by the $\Omega_{\mathrm{z}}$. Interestingly. along line 15 , the $\mathrm{W}$ profile is shows greater values when compared with non-rotating case, indicating a redistribution of the flow downstream from the pedestal before reaching the exit section.

In Figure 9, the velocity profiles along two lines obtained by intersection of plane 0 with planes 2 and 3 are shown. Plane 0 is at middle of the inlet section (in y direction) and then it gives information about the flow where the influence of the two walls is minimum. Along line 02 , the $\mathrm{U}$ velocity has a lower value in the first portion of the line (where $\mathrm{z}$ ranges between 0.0 and $0.15)$. This is due to the shape of the recirculation region originating immediately downstream of the corner. Along line 03 the $U$ velocity is not affected by rotation and the profiles are similar in the two cases. On the contrary, W velocity is damped on plane 0 showing smaller values everywhere. The velocity magnitude plots confirm such behaviour.

The occurrence of unsteadiness in the flow is now investigated. In Figure 10, the FFT of the velocity fluctuations 
computed in a number of monitoring point in the non-rotating and rotating case are shown.

The black line identify the main frequency that correspond to the presence of a strong unsteadiness affecting the entire domain. The value of this period is slightly different in the two cases. In particular, in the rotating case the fluctuation period has a smaller value (see Table 4). Assuming as reference the average pedestal height and the inlet bulk velocity, we obtained a Strouhal number of 0.22 for the non-rotating case and of 0.3 for the rotating one. On the other hand, the unsteadiness induced by the rotation is vigorous and with huge amplitude. In particular, in the wedge region upstream of the pedestals, the flow is subjected to a charge-discharge motion, much more pronounced than in the non-rotating case (see Figure 11).
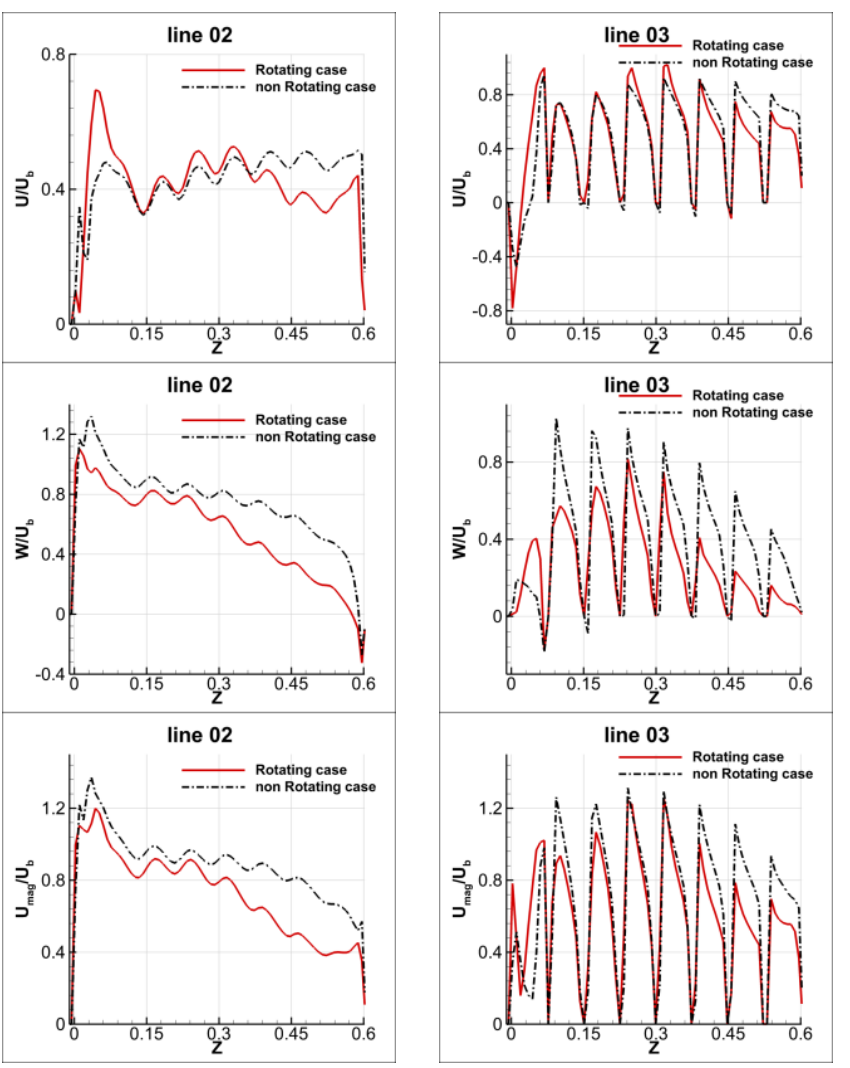

Figure 9. Comparisons of velocity components profiles for two different lines obtained by the intersection of 0 plane with 2 and 3 planes.
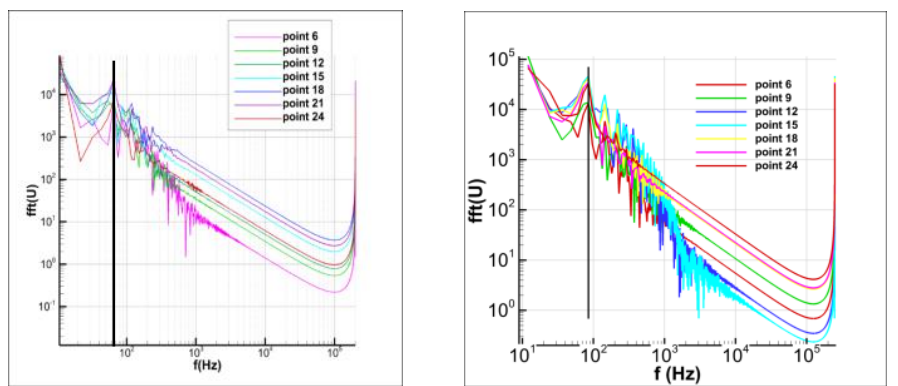

Figure 10. Comparisons of velocity components profiles for two different lines obtained by the intersection of 0 plane with 2 and 3 planes.

The charge/discharge motion affects also the motion in the pedestal region. When charging (i.e. pushing huge flow quantity on the top of the wedge - Figure 11 left) the mass flow in the region between pedestals 4 and 7 is strongly increased. In the opposite situation, mass flow is concentrated in the region between the lower wall and pedestal 4 .

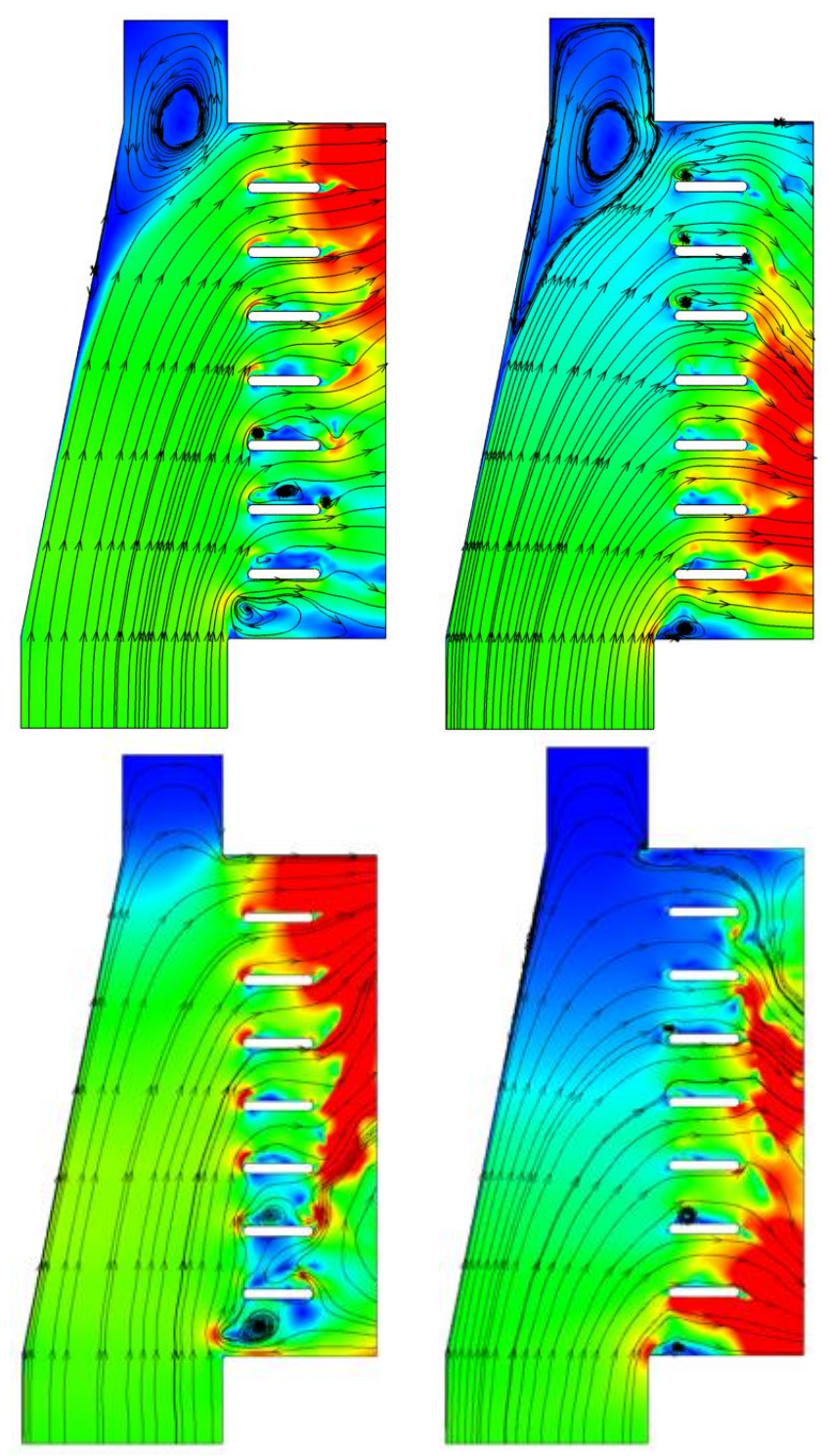

Figure 11. Realisations of velocity magnitude plots on plane 1. Top: non-rotating; bottom: rotating. Realisations refer to opposite phases of the period.

The strong amplitude of velocity fluctuation is also confirmed by the plot of turbulent kinetic region, whose production term is strongly dependent from the velocity gradients and form the shear flow. 
Table 4: Unsteadiness period

\begin{tabular}{ccc}
\hline & Non-rotating & Rotating \\
\hline Period & 0.019 & 0.011 \\
\hline Strohual & 0.22 & 0.30 \\
\hline
\end{tabular}

As shown in Figure 12, the turbulent kinetic energy distribution downstream from the pedestals show a wide spreading with higher values. Furthermore, also the peak in the upper part of the pedestals is more pronounced due to the strong shear occurring also upstream.
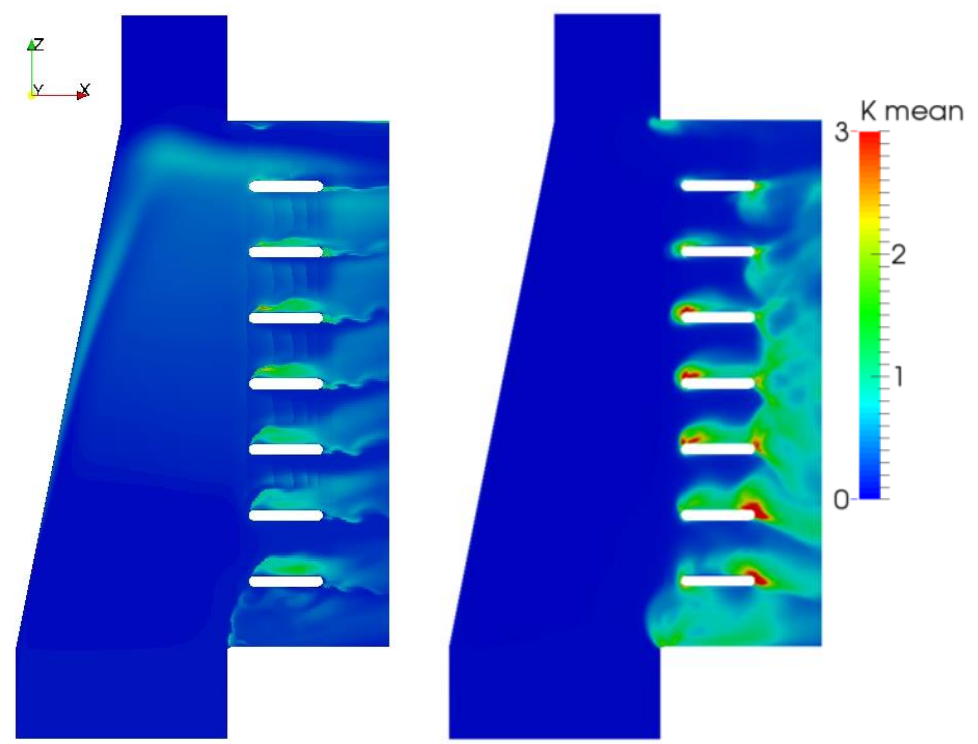

Figure 12. Turbulent kinetic energy plots on plane 1. Left: nonrotating; right: rotating.

\section{Particles}

Results will be discussed referring to the view plans reported in Figure 13.

Figure 14 shows some trajectories of the non-rotating and rotating case. Comparing the two cases, the strong effect of the large recirculation region at the closed tip of the channel (see also Figure 11) is put in evidence. In the non-rotating case (Figure 14 left) particles are not only entrapped by the recirculation bubble, but since it affects the whole flow field, particles are more spread, especially in the upper half of the channel. In fact, in rotating case (Figure 14-right) the recirculation bubble is weaker and particles entrapped in it do not recirculate, and stay more clustered.

Another difference in particle trajectories concerns the pedestal region. As shown in Figure 14 left, in non-rotating case particles hardly impact the leading edge of pedestals, rather impacting their top and bottom surfaces, and then bouncing off. On the contrary, in rotating case many particles impact the leading edge of pedestals and surrounding regions, then rebound. This leads to a large number of rebounding particles undergoing other impacts on different pedestals.
A better visualization of the effect of the two different flow fields on particle motion is provided by Figure 15 and Figure 16, which show the impact points coloured by impact angles. Rotation promotes the flow exit, thus particles undergo a smaller number of impacts (Figure 16) comparing with the nonrotating situation (Figure 15). The effect of rotation is more evident at the tip. In the non-rotating case a large number of particles entrapped by the recirculation bubble are pushed to the tip walls, impacting and eroding them as shown in Figure 15 (view plans D and E, dashed ellipses). These impact points are completely absent in the rotating case (Figure 16, same view plans) since it does not form any recirculation bubble.

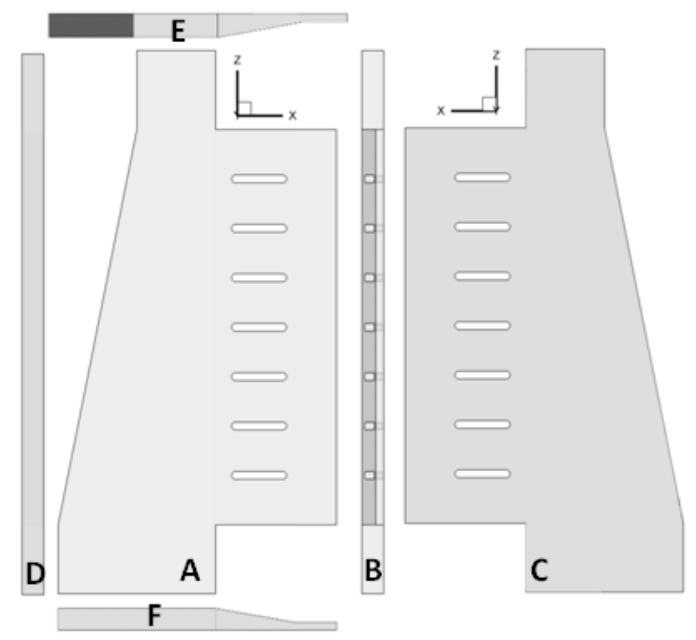

Figure 13. Reference view planes (A-F).

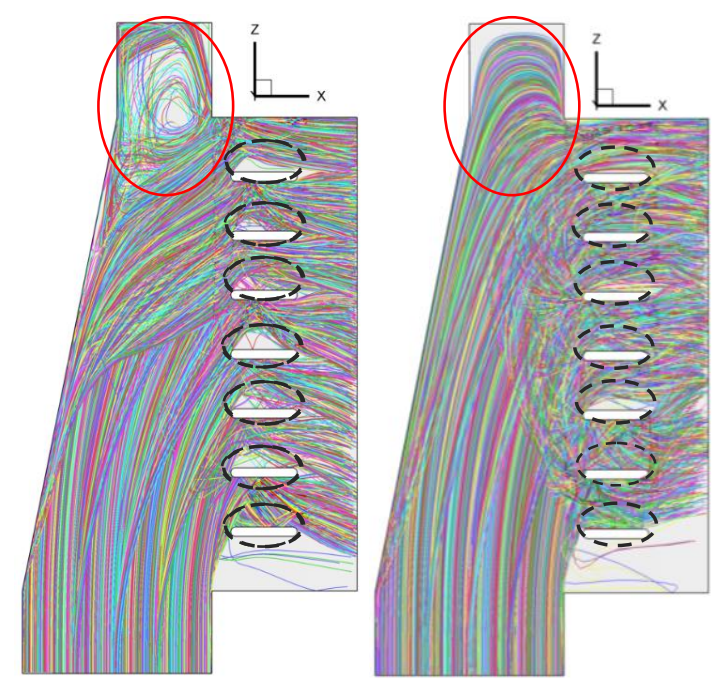

Figure 14. Trajectories of some particles in non-rotating (left) and rotating (right) case (view plane A).

Another aspect that is worth to note, is the impact of a number of particles in the region just below the tip on both sides of the channel (Figure 15, view plans A and C, black ellipses). These impacts are due to the interaction between the main flow and the recirculation bubble at the tip, which spreads 
the entrained particles and makes them hit the side walls. Both these erosion zones are missing in the rotating case (Figure 16, same view plans), where impacts in this region are mostly clustered near the pedestals.

Lastly it is interesting to put the attention on the impact lines developing in both view plans $\mathrm{A}$ and $\mathrm{C}$, starting from the connection between the inlet and the wedge-shaped regions (line-dot-line ellipses in Figure 15and Figure 16). They are due to the small recirculation caused by the joint between these two regions (Figure 17), and entrap particles mainly entering the domain from section S1 (Figure 4), then pushing them toward the side walls.

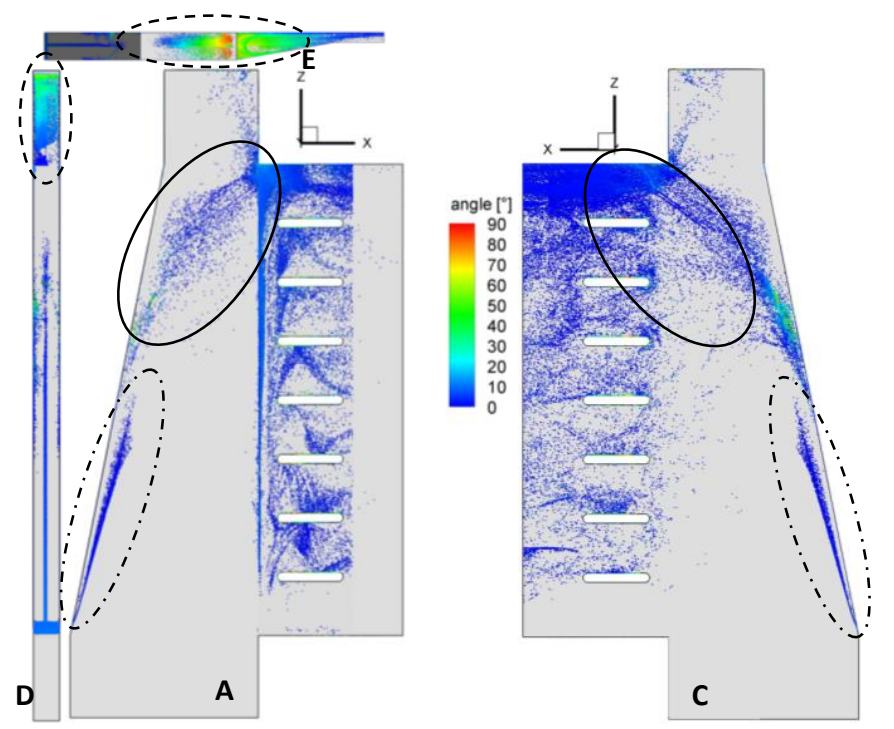

Figure 15. Impact angles in non-rotating case.

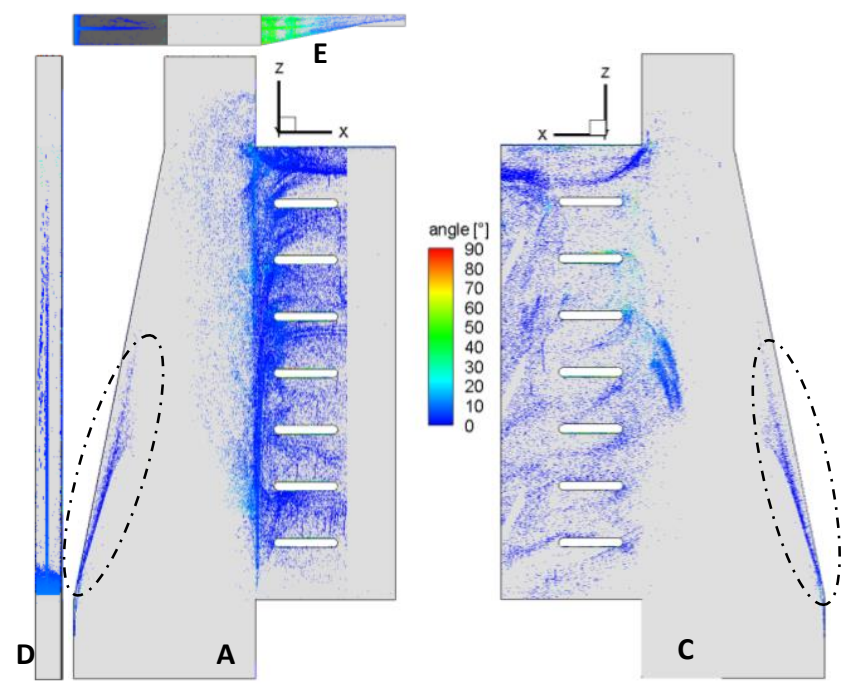

Figure 16. Impact angles in rotating case.

All these effects have an impact on the erosion patterns, reported in Figure 18 (non-rotating case) and Figure 19 (rotating case). Erosion in non-rotating channel involves regions different from those of the rotating case. Larger differences are related to the absence, in the rotating-case, of the large recirculation bubble at the tip of the channel, and to the impact of particles on the leading edges of pedestals. As said before, it affects not only the motion of particles within the recirculation (in non-rotating case) but also those at the interaction with the main stream.

Erosion patterns on pedestal surfaces, are reported in Figure 20 and Figure 21. As shown in figures, erosion on both surfaces of pedestals is more pronounced in the rotating case. As a matter of fact the different interaction between particles and pedestals in the two cases, and the larger velocity induced by rotation, result in a larger number of impact on pedestal surface in rotating case. Bottom surfaces are the most exposed to erosion, being pedestals $\mathrm{P} 1$ and $\mathrm{P} 7$ the less eroded ones.

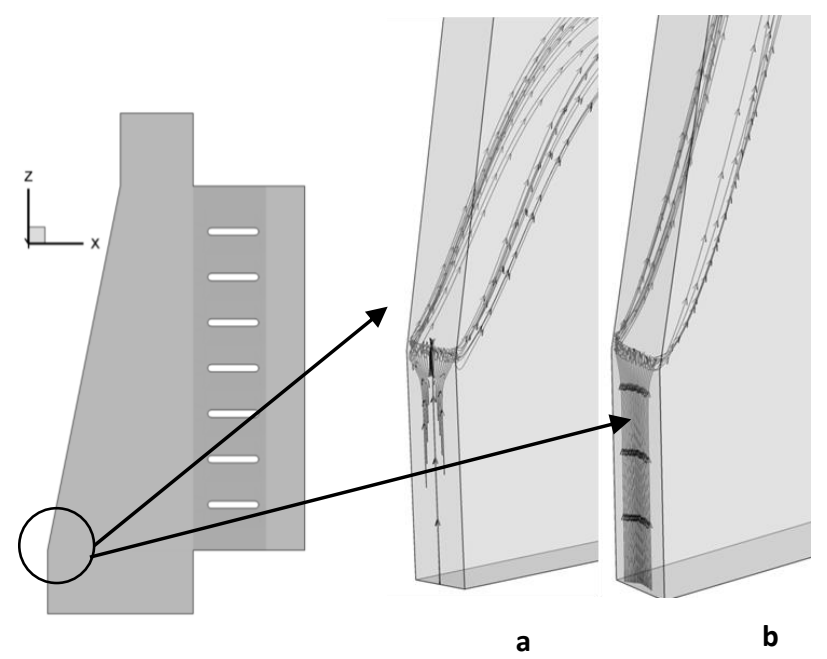

Figure 17. Recirculation forming at the joint between inlet and wedge-shaped regions: a) non-rotating and b) rotating case.

\section{CONCLUSIONS}

Prediction of the particle-laden flow in the internal cooling channel of a rotating gas turbine blade (in the trailing edge region) was discussed. This analysis allows to obtain information about the erosion mechanisms inside a region that is hardly accessible by experiments.

To account for the influence of rotation, the authors derived a correction of the non-linear $\zeta$-f model and demonstrated its effectiveness.

The rotational effects strongly changed the flow behaviour inside the channel leading to a vigorous charge/discharge mechanism and altering the period and amplitude of flow unsteadiness.

The change in the flow directly influenced the particles trajectories and erosion of the solid surfaces. The absence of recirculation on the tip region reduces the impacts in such region. Besides, rotation pushes particles toward the exit, thus forcing them to impact the leading edge region of pedestals.

It is worth noting the two erosion lines due to the small recirculation developing at the joint between inlet and wedge- 
shaped regions: this recirculation is weakly affected by rotation, thus these eroded regions are present in both the cases.

The final result of all the combined effects induced by rotation is a completely different erosion pattern in the two studied cases: in the latter case, erosion is mostly focused on the top of the channel (view plan E), and on the side C, especially on the pedestal region (mainly in the upper part) and at the outlet. On the contrary, in rotating case erosion in more evident in the view plan A (pedestal region and before), and in the view plan $\mathrm{C}$ (but on middle of pedestal region, and at the outlet).
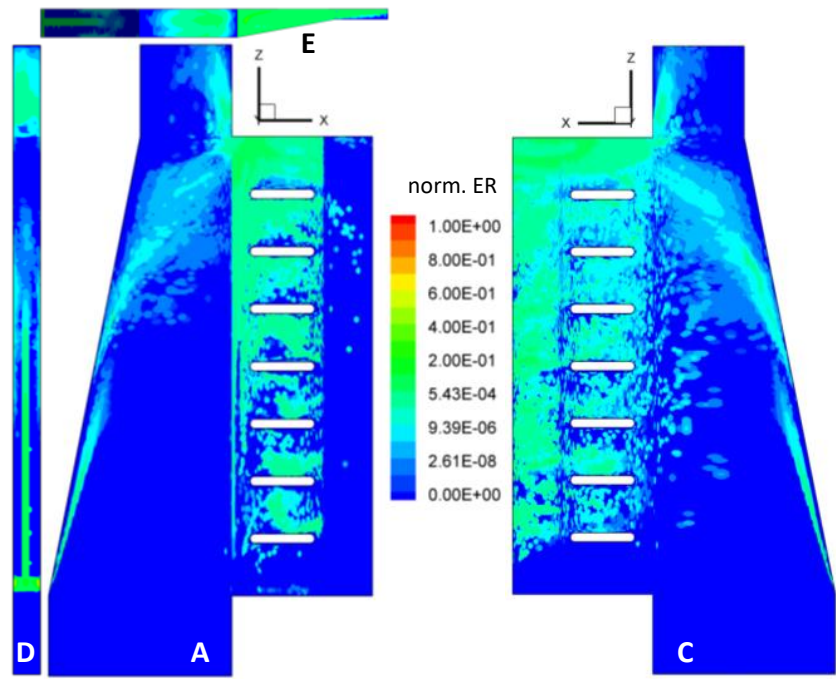

Figure 18. Normalized erosion rate in non-rotating case.
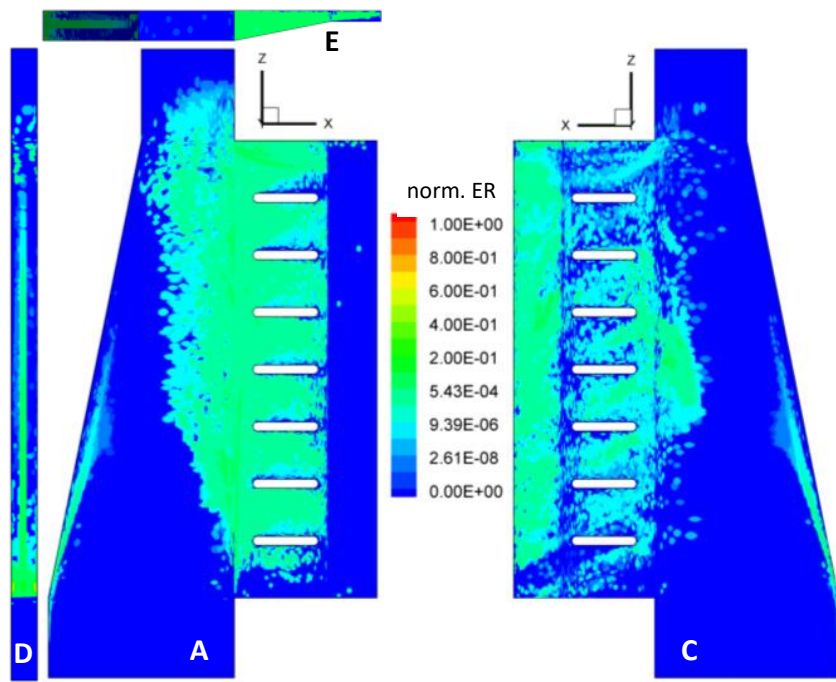

Figure 19. Normalized erosion rate in rotating case.

\section{ACKNOWLEDGMENTS}

Part of the computing resources and the related technical support used for this work have been provided by CRESCO/ENEAGRID High Performance Computing infrastructure and its staff; see http://www.cresco.enea.it for information. CRESCO/ENEAGRID High Performance
Computing infrastructure is funded by ENEA, the Italian National Agency for New Technologies, Energy and Sustainable Economic Development and by national and European research programs.
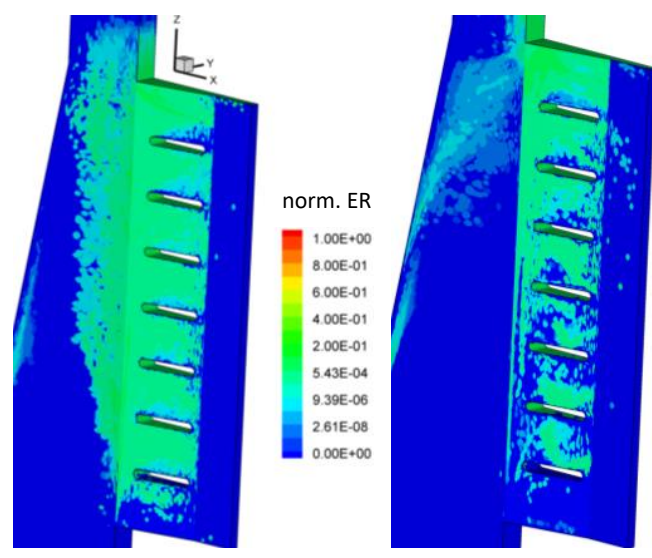

Figure 20. Normalized erosion rate on the bottom surface of pedestals, in rotating (left) and non-rotating (right) case.
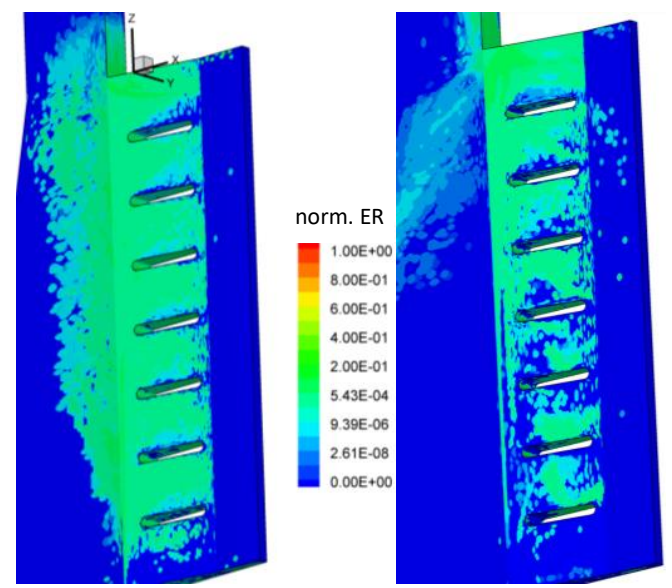

Figure 21. Normalized erosion rate on the upper surface of pedestals, in rotating (left) and non-rotating (right) case.

\section{REFERENCES}

[1]. Bianchini, B., Simonetti, F., Tarchi, L., Facchini, B., Zecchi, 2012. "Numerical and experimental investigation of turning flow effects on innovative pin fin arrangements for trailing edge cooling configurations", Journal of Turbomachinery Vol. 134, Iss.2, 021005 doi:10.1115/1.4003230.

[2]. Chaouat B., 2012, "Simulations of turbulent rotating flows using a subfilter scale stress model derived from the partially integrated transport modeling method", Physics Of Fluids 24, 045108.

[3]. Tafti, D. K., 2005, Evaluated the role of subgrid stress modelling in a ribbed duct for the internal cooling of 
turbine blades, Int. J. Heat and Fluid Flow, Vol. 26, pp. 92-104.

[4]. Borello D., Rispoli F., Salvagni A., Hanjalic K., 2014, "Effects of Rotation on Flow in a Rib-roughened Channel: LES Study", ETMM10, September 17-19, Marbella, Spain (selected for publication on IJHFF).

[5]. Donahoo, E. E., Kulkarni, A. K., Belegundu, A. D., and Camci, C., 2001. "Determination of Optimal Row Spacing for a Staggered Cross-Pin Array in a Turbine Blade Cooling Passage". J. Enhanced Heat Transfer vol. 8, pp. 41-53.

[6]. Hamilton, L. J., Adametz, D. S., Lind, E. K., and Gropinath, A., 2002. "Numerical Analysis of the Performance of a Staggered Cross-Pin Array Heat Exchanger”. The Eighth AIAA/ASME Joint Thermophysics and Heat Transfer Conference.

[7]. Borello D., Capobianchi P., De Petris M., Rispoli F., Venturini P., 'Unsteady RANS analysis of particles deposition in the coolant channel of a gas turbine blade using a non-linear model', Turbo Expo 2014, Dusseldorf.

[8]. Petterson Reif B.A., Durbin P., Ooi, A., 1999, "Modeling rotational effects in eddy-viscosity closures", Int. J. Heat and Fluid Flow, pp. 564-572.

[9]. Venturini P., Borello D., Hanjalić K., Rispoli F, 2012, "Modelling of particle deposition in an environment relevant to solid fuel boilers", Applied Thermal Engineering 49, pp. 131-138.

[10]. D. Borello, F. Rispoli, P. Venturini, 2012, An integrated particle-tracking impact/adhesion model for the prediction of fouling in a subsonic compressor, ASME Journal of Engineering for Gas Turbine and Power, 2012;134(9).

[11]. Tabakoff W., Kotwal R., Hamed A., 1979, Erosion study of different materials affected by coal ash particles, Wear, 52, pp. 161-173.

[12]. Borello D., Delibra G., Andreini A., Bianchini C., 2012, "Unsteady CFD analysis of turbulent flow and heat transfer in a gas turbine blade trailing edge subjected to rotation", ASME Turbo Expo 2012, Copenhagen, Denmark, paper no. GT2012-69903.

[13]. Kristoffersen R., Andersson, H.I., 1993, Direct simulations of low-Reynolds-number turbulent flow in a rotating channel, Journal of Fluid Mechanics, pp. 163-197.
[14]. Sommerfeld M., van Wachem B., Oliémans R., 2009, "Dispersed turbulent multi-phase flow. Best practice guidelines", ERCOFTAC.

[15]. Crowe C.T., Mechaelides E.E., 2006, "Basic concepts and definitions", in Multiphase Flow Handbook (C.T. Crowe), Boca Raton.

[16]. Hamed A., 1998, "Effect of particle characteristics on trajectories and blade impact", ASME Journal of fluids Engineering, vol.110, 33-37.

[17]. Seggiani M., Bardi A., Vitolo S., 2000, "Prediction of fly-ash size distribution: a correlation between the char transition radius and coal properties", Fuel, 79, 999. 1002.

[18]. Crowe C.T., Troutt T.R., Chung J. N., 1996, "Numerical Models for two-phase turbulent flows," Annu. Rev. Fluid Mech., 28, pp. 11-43.

[19]. Armenio V., and Fiorotto V., 2001, "The Importance of the forces acting on particles in turbulent flows," Phys. Fluids, 13(8), pp. 2437-2440.

[20]. Anielli D., Borello D., Rispoli, F., Salvagni A., Venturini P., 2015, "Prediction of particle erosion in the internal cooling channels of a turbine blade", European Turbomachinery Conference, Madrid, Spain, April 2015.

[21]. Borello D., D’Angeli L., Salvagni A., Venturini P., Rispoli F., Study of particle deposition in gas turbine blades in presence of film cooling, ASME Turbo Expo 2014, Dusseldorf, Germany, paper no. GT2014-26250.

[22]. Venturini P., Borello D., Hanjalić K., Rispoli F, 2012, "Modelling of particle deposition in an environment rele-vant to solid fuel boilers", Applied Thermal Engineering 49, pp. 131-138.

[23]. A. Corsini, F. Rispoli, P. Venturini, G. Sheard: Numerical simulation of coal-fly ash erosion in an induced draft fan. ASME Turbo Expo 2012: Power for Land, Sea and Air (paper GT2012-69048), June 11-15, 2012, Copenhagen, Denmark. 